Louisiana State University

LSU Digital Commons

3-10-2008

\title{
Host galaxies catalog used in LIGO searches for compact binary coalescence events
}

\author{
Ravi Kumar Kopparapu \\ Louisiana State University \\ Chad Hanna \\ Louisiana State University \\ Vicky Kalogera \\ Northwestern University \\ Richard O'Shaughnessy \\ Pennsylvania State University \\ Gabriela González \\ Louisiana State University
}

See next page for additional authors

Follow this and additional works at: https://digitalcommons.Isu.edu/physics_astronomy_pubs

\section{Recommended Citation}

Kopparapu, R., Hanna, C., Kalogera, V., O'Shaughnessy, R., González, G., Brady, P., \& Fairhurst, S. (2008). Host galaxies catalog used in LIGO searches for compact binary coalescence events. Astrophysical Journal, 675 (2), 1459-1467. https://doi.org/10.1086/527348 


\section{Authors}

Ravi Kumar Kopparapu, Chad Hanna, Vicky Kalogera, Richard O'Shaughnessy, Gabriela González, Patrick R. Brady, and Stephen Fairhurst 


\title{
HOST GALAXIES CATALOG USED IN LIGO SEARCHES FOR COMPACT BINARY COALESCENCE EVENTS
}

\author{
Ravi Kumar Kopparapu, ${ }^{1,2,3}$ Chad Hanna, ${ }^{2}$ Vicky Kalogera, ${ }^{4}$ Richard O’Shaughnessy, ${ }^{3,4}$ \\ Gabriela González, ${ }^{2}$ Patrick R. Brady, ${ }^{5}$ and Stephen Fairhurst $5,6,7$ \\ Received 2007 June 7; accepted 2007 November 21
}

\begin{abstract}
An up-to-date catalog of nearby galaxies considered to be hosts of binary compact objects is provided, with complete information about sky position, distance, extinction-corrected blue luminosity, and error estimates. With our current understanding of binary evolution, rates of formation and coalescence for binary compact objects scale with massive-star formation, and hence the (extinction-corrected) blue luminosity of host galaxies. Coalescence events in binary compact objects are among the most promising gravitational-wave sources for ground-based gravitationalwave detectors such as LIGO. Our catalog and associated error estimates are important for the interpretation of analyses carried out for LIGO, in constraining the rates of compact binary coalescence, given an astrophysical population model for the sources considered. We discuss how the notion of effective distance, created to account for the antenna pattern of a gravitational-wave detector, must be used in conjunction with our catalog. We also note that the catalog provided can be used in other astronomical analysis of populations that scale with galaxy blue luminosity.
\end{abstract}

Subject headings: binaries: close — catalogs — galaxies: luminosity function, mass function gravitational waves - stars: neutron

Online material: machine-readable table

\section{INTRODUCTION}

Compact binary coalescence (CBC) events, such as neutron star or black hole mergers, are one of the primary gravitationalwave sources for ground-based interferometers such as LIGO. ${ }^{8}$ LIGO's third (S3, 2003 October 31-2004 January 9) and fourth (S4, 2005 February 22-March 23) science runs have reached significant extragalactic distances (Abbott et al. 2007) into the nearby universe. Especially for massive compact binaries whose components are black holes, the range extends beyond the Virgo Cluster. Interpreting searches for signals from compact binary coalescence in the LIGO data sets requires some information about putative binary compact object populations in known nearby galaxies, as well as some sense of how the population scales at larger distances. The nearby galaxy catalog discussed here is representative of the distribution of such extragalactic populations, and the procedures described here are such as are used for LIGO data analysis, such as assigning astrophysically meaningful upper limits given nondetection. An accurate upper limit that correctly incorporates our best information about galaxy distributions requires a model of the nearby overdense region, since the current LIGO network's range probes this overdensity.

Binary compact objects are usually produced via the evolution of massive stellar binaries. Since short-lived massive stars emit more blue light than all other stars in a galaxy combined, blue light is a well-known tracer of star formation in general and the

\footnotetext{
${ }^{1}$ Center for Computation and Technology, Louisiana State University, Baton Rouge, LA 70803.

2 Department of Physics and Astronomy, Louisiana State University, Baton Rouge, LA 70803.

3 Current address: Center for Gravitational Wave Physics, 104 Davey Lab, Pennsylvania State University, University Park, PA 16802-6300.

4 Department of Physics and Astronomy, Northwestern University, 2145 Sheridan Road, Evanston, IL 60208.

5 Department of Physics, University of Wisconsin-Milwaukee, P.O. Box 413, Milwaukee, WI 53201.

${ }^{6}$ School of Physics and Astronomy, Cardiff University, Cardiff, CF2 3YB, UK.

7 LIGO Laboratory, California Institute of Technology, Pasadena, CA 91125.

8 See http://www.ligo.org.
}

birthrate of these massive stars in particular. Given the short lifetimes of the known Milky Way double compact object population and the slow rate of change in star formation expected in nearby and distant galaxies, Phinney (1991) has argued that a galaxy's blue luminosity should linearly scale with its compact binary coalescence rate.

The sensitivity of LIGO to compact binary coalescence signals depends on the distance and sky position of the coalescence event, and therefore the distribution of known nearby galaxies in blue luminosity and in space is the minimum information needed to properly interpret searches of the LIGO data sets.

It is possible that compact binary populations that are not related to regions of star formation may exist in the universe. A mass, metallicity, and morphology dependent star formation history may be needed to account for these populations. ${ }^{9}$ Nevertheless, the work described here is limited to the blue-light luminosity as a tracer of the compact binary population.

The contribution of elliptical galaxies to the merger rates is potentially significant beyond the Virgo cluster (de Freitas Pacheco et al. 2006), whereas their blue luminosity is not representative of their putative compact binary populations. However, at large distances, the fractional blue luminosity produced in ellipticals is about 10\% (Driver \& Allen 2007), and at short distances the contribution is negligible, because there are fewer ellipticals in the nearby local universe. De Freitas Pacheco et al. (2006) conclude that the event rate for an elliptical galaxy with the same blue luminosity as a spiral galaxy is a factor of 5 times larger on average. We conclude that LIGO rate upper limits derived from the catalog presented here would change by less than a factor of 1.5 due to a correction for elliptical galaxies.

Our blue light census will also implicitly not account for any potential contribution from globular clusters to the compact binary coalescence rate of the nearby universe. Phinney (1991) has

\footnotetext{
${ }^{9}$ Lipunov et al. (1995) adopts a mass normalization to derive their event rate of 1 per year within $50 \mathrm{Mpc}$ using an older version of Tully's catalog, whereas we use a blue-light normalization and the up-to-date Tully catalog $(\S 2)$. We also consider issues such as the antenna pattern of the detector and completeness corrections.
} 
TABLE 1

Compact Binary Coalescence Galaxy (CBCG) Catalog

\begin{tabular}{|c|c|c|c|c|c|c|}
\hline Name & R.A. & Decl. & $\begin{array}{c}D \\
(\mathrm{kpc})\end{array}$ & $L^{\mathrm{a}}$ & $\sigma_{\mathrm{mag}}$ & $\sigma_{D}$ \\
\hline Sagitt & 1855.10 & 3032.40 & 24.0 & 0.001 & 0.30 & 0.10 \\
\hline LMC ..................... & 0523.30 & -6945.20 & 49.0 & 0.098 & .30 & 0.10 \\
\hline $0524-69 \ldots \ldots \ldots$ & 0524.00 & -6900.00 & 50.0 & 0.325 & 0.38 & 0.10 \\
\hline SMC ……................ & 0052.40 & -7249.40 & 58.0 & 0.039 & 0.30 & 0.10 \\
\hline $0051-73 \ldots \ldots \ldots$ & 0051.00 & -7300.00 & 60.0 & 0.067 & 0.38 & 0.10 \\
\hline Sextans B ........... & 1000.00 & +0519.50 & 100.0 & 0.002 & 0.30 & 0.10 \\
\hline Fornax ……............. & 0239.50 & -3426.50 & 138.0 & 0.001 & 0.30 & 0.10 \\
\hline NGC $6822 \ldots \ldots . .$. & 1944.90 & -1449.00 & 490.0 & 0.006 & 0.38 & 0.10 \\
\hline NGC $0185 \ldots \ldots . .$. & 0039.00 & +4820.00 & 620.0 & 0.006 & 0.38 & 0.10 \\
\hline UGL $192 \ldots \ldots \ldots . .$. & 0020.38 & +5917.59 & 660.0 & 0.047 & 0.42 & 0.10 \\
\hline
\end{tabular}

Note.-Table 1 is published in its entirety in the electronic edition of the Astrophysical Journal. A portion is shown here for guidance regarding its form and content.

${ }^{\text {a }}$ In units of $1 \times 10^{10} L_{B, \odot}$.

argued that the contribution of globular clusters to double neutron star mergers in the Galaxy would not exceed $10 \%$ of the coalescence rate due to the Galactic field. On the other hand, it has been argued that the contribution of globular clusters to binary black hole coalescence may be very significant (see, e.g., Portegies Zwart \& McMillan 2000; O’Leary et al. 2007). However, these cluster contributions are expected to become significant at distances beyond the Virgo Cluster, where a more significant fraction of ellipticals with large globular cluster systems will eventually enter LIGO's detection volume.

We have used mostly publicly available astronomical catalogs of galaxies to compile a catalog used in the S3/S4/S5 (fifth science run, ${ }^{10} 2005$ November 4 -present) LIGO data set analyses. We discuss the methodology used to compile this galaxy catalog and briefly describe how this information feeds into LIGO rate estimates. In $\S 2$, we describe all the elements involved in compiling the galaxy catalog and assessing the relevant errors and uncertainties. In $\S 3$, we derive a correction factor to account for incompleteness in the catalog, guided also by the blue-light volume density estimated from the Sloan Digital Sky Survey and earlier surveys. In $\S 4$, we discuss how the corrected catalog and resulting blue light distribution as a function of distance is used to bound the rate of compact binary coalescence using data from the recent LIGO science runs. If the maximum distance to which a search could detect a compact binary coalescence is known, then the expected number of detectable events can be derived. Some concluding remarks are made in $\S 5$.

\section{COMPILATION OF GALAXY CATALOG}

We have compiled a catalog, ${ }^{11}$ the Compact Binary Coalescence Galaxy (CBCG) catalog, of nearby galaxies which could host compact binary systems. For each galaxy out to $100 \mathrm{Mpc}$, the catalog provides the equatorial coordinates, distance to the galaxy, and the blue luminosity corrected for absorption. Estimates of the systematic errors on distance and luminosity are also provided (see Table 1).

The CBCG catalog is compiled from information provided in the following four astronomical catalogs: (1) the Hubble Space Telescope (HST) Key Project catalog used to measure the Hubble constant (Freedman et al. 2001), (2) Mateo's dwarf galaxies of the

\footnotetext{
10 See http://1hocds.ligo-wa.caltech.edu/ 11_news/s5_news/s5article.htm.

11 See http://www.lsc-group.phys.uwm.edu/cgi-bin/cvs/viewcvs.cgi/checkout/ lalapps/src/inspiral/inspsrcs100Mpc.errors?cvsroot=lscsoft.
}

local group catalog (Mateo 1998), (3) the HyperLeda (LEDA) database of galaxies (Paturel et al. 2003), ${ }^{12}$ and (4) an updated version of the Tully Nearby Galaxy Catalog (B. Tully 2006, private communication).

When combining these catalogs, distances and luminosities reported in the HST, Mateo, and Tully catalogs were generally adopted over those in the LEDA catalog, since these catalogs use more accurate distance determination methods than LEDA. Nevertheless, LEDA served as the baseline for comparisons in the range $10-100 \mathrm{Mpc}$, since it is the most complete.

\subsection{Distances}

One of the primary objectives of the HST Key Project was to discover Cepheid variables (stars with periodic variations in brightness) in several nearby spiral galaxies and measure their distances accurately using the period-luminosity relation for Cepheids. Cepheid distance determination to nearby galaxies is one of the most important and accurate primary distance indicators. The distance information from the HST Key Project is considered to be the most accurate in the CBCG catalog; there are 30 galaxies in our catalog for which we adopt distances from the HST Key Project.

Mateo's review (Mateo 1998) of properties of the dwarf galaxies in the Local Group provides distance and luminosity information for each galaxy considered. Since the parameters in this catalog were derived from focused studies on each individual galaxy, we consider it the most accurate next to the HST measurements for nearby galaxies. Moreover, it has reasonably comprehensive information on the Local Group's dwarf galaxies; there are 18 sources in the CBCG catalog which adopt distances (and luminosities) from Mateo's compilation.

It becomes increasingly difficult to use primary distance estimators such as Cepheid stars in more distant galaxies. Therefore, secondary distance methods are used to measure larger distances. Tully's catalog has up to three types of distances for each source:

1. Quality distance $\left(D_{Q}\right)$ is based on either Cepheid measurements, surface brightness fluctuations, or the tip of the red giant branch. There are 409 galaxies with such a distance in the CBCG catalog.

2. H I luminosity-line-width distances $\left(D_{\mathrm{H}_{\mathrm{I}}}\right)$ are obtained from the Tully-Fisher relation, where the maximum rotational velocity of a galaxy (measured by the Doppler broadening of the $21 \mathrm{~cm}$ radio emission line of neutral hydrogen) is correlated with the luminosity (in the $B, R, I$, and $H$ bands) to find the distances. There are 553 galaxies in the catalog with such a distance.

3. Model distance $\left(D_{M}\right)$ is derived from an evolved dynamical mass model that translates galaxy radial velocities into distances. This model is an update of the least action model described by Shaya et al. (1995) and takes into account the deviations from a perfect Hubble flow due to a spherically symmetric distribution of mass centered on the Virgo Cluster.

All galaxies have a calculated model distance. Whenever available, $D_{Q}$ distances are preferred due to their smaller uncertainties, then the $D_{\mathrm{H}_{\mathrm{I}}}$, followed by $D_{M}$.

The remaining galaxies come from LEDA, which does not provide distances explicitly, but instead provides measured radial velocities corrected for infall of the Local Group toward the Virgo cluster $\left(v_{\text {vir }}\right)$. We obtain the LEDA distance $\left(D_{L}\right)$ using Hubble's law with the Hubble constant $H_{0}=73 \mathrm{~km} \mathrm{~s}^{-1} \mathrm{Mpc}^{-1}$ reported by Spergel et al. (2007). Although corrections to the recessional

\footnotetext{
12 Available at http://leda.univ-lyon1.fr/intro.html.
} 

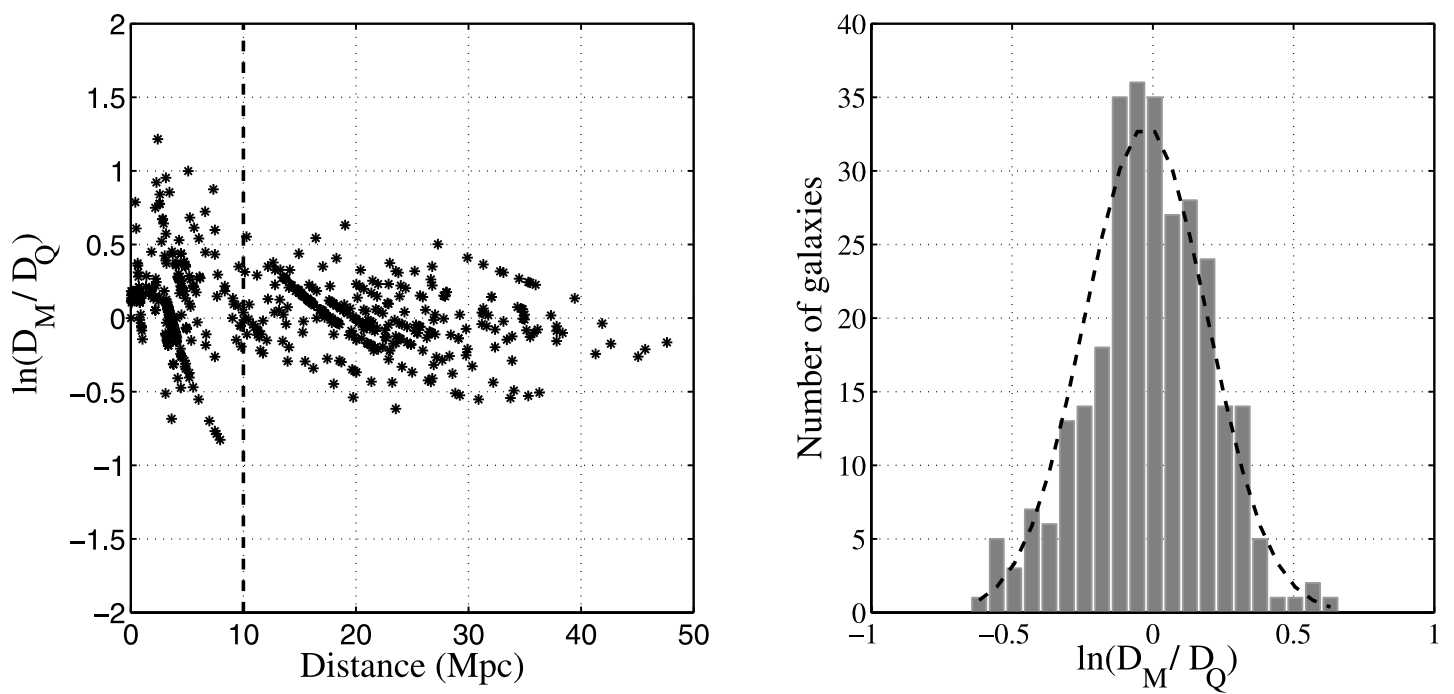

FIG. 1.- In order to obtain reasonable estimates for Tully's model distances, we compare galaxies that have values for both. We only consider galaxies beyond 10 Mpc, since model distances and LEDA distances are not reliable below this value. All galaxies below $10 \mathrm{Mpc}$ have better distance estimates. The Tully quality distance has roughly a 0.1 logarithmic error. The best-fit Gaussian for $\ln \left[D_{M} / D_{Q}\right]$ implies a fractional error $\sigma$ of $0.24 \mathrm{in} \log$. Subtracting these uncertainties in quadrature gives an error of 0.22 for Tully model distances.

velocity were made, this method of calculating distances is still highly uncertain. Hence, we use Hubble's law to evaluate the distances only to the galaxies for which $v_{\mathrm{vir}} \geq 500 \mathrm{~km} \mathrm{~s}^{-1}(7 \mathrm{Mpc})$ and peculiar velocities are expected to be more of a perturbation.

The error in a distance depends strongly on the method used to measure that distance. The HST sources, although a small contribution to the galaxy catalog, have the smallest errors $(<10 \%$; Freedman et al. 2001). The three different distance methods in Tully's catalog have different errors. The quality distance $D_{Q}$ also has a low error (10\%), followed by the $\mathrm{H}$ I luminosity-linewidth distance $D_{\mathrm{H}_{\mathrm{I}}}(20 \%)$. To obtain an estimate for the errors of $D_{M}$, we compare them with $D_{Q}$ for the set of galaxies that have both types of distance estimates. The best-fit Gaussian (see Fig. 1) to the logarithm of fractional errors has a $1 \sigma$ width of 0.24 , which, when subtracted in quadrature with $D_{Q}$ error, gives a 0.22 distance error associated with $D_{M}$.

Because errors in $v_{\text {vir }}$ are not given in LEDA, we follow a similar procedure to find LEDA distance errors, $D_{L}$. We compare the calculated $D_{L}$ with $D_{Q}$ for galaxies in both catalogs to obtain

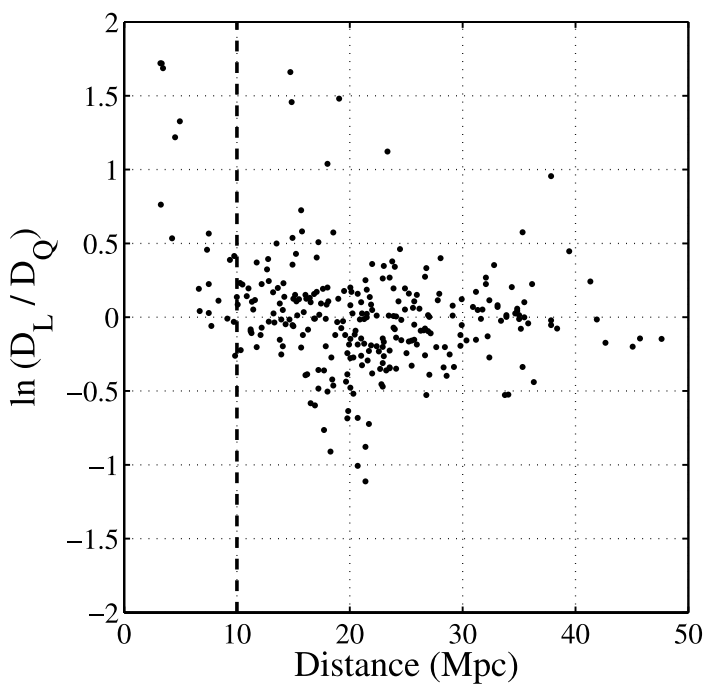

uncertainty estimates in $D_{L}$. The plot in Figure 2 shows the bestfit Gaussian to the logarithm of fractional errors with a $1 \sigma$ width of 0.27 , which, subtracted in quadrature with $D_{Q}$ distance errors, gives a total distance error of $0.25 .^{13}$

\subsection{Blue Luminosities}

The distribution of binary compact objects in the nearby universe is expected to follow the star formation in the universe, and a measure of star formation is the blue luminosity of galaxies corrected for dust extinction and reddening (Phinney 1991). Hence, for each galaxy, we calculate the blue luminosity $L_{B}$ from the absolute blue magnitude of the galaxy $M_{B}$ (corrected for internal and Galactic extinctions). For convenience, blue luminosity is provided in units of $L_{10} \equiv 10^{10} L_{B, \odot}$, where $L_{B, \odot}=2.16 \times$ $10^{33} \mathrm{ergs} \mathrm{s}^{-1}$ is the blue solar luminosity derived from the blue

13 For searches of the S3 and S4 LIGO data (Abbott et al. 2007), with smaller ranges, a more conservative uncertainty of $40 \%$ was used for LEDA distances.

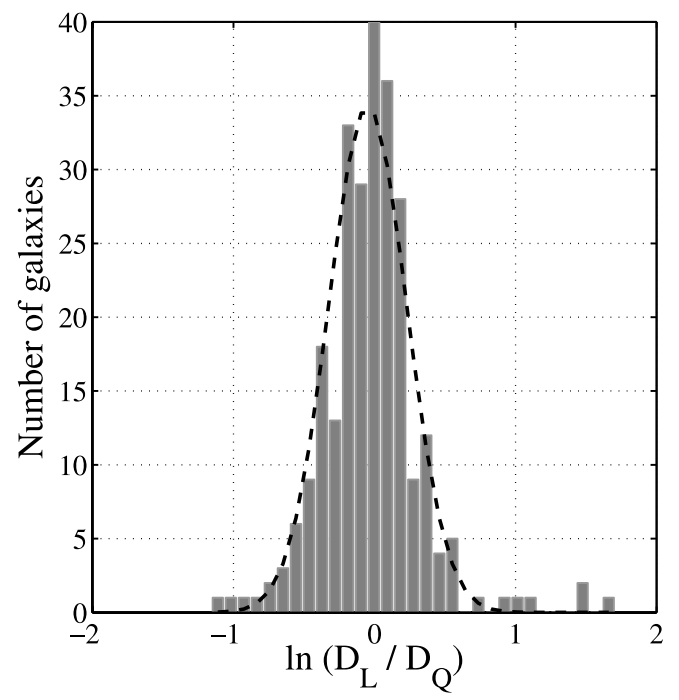

FIG. 2.-Fractional error analysis as in Fig. 1 for LEDA distances. By comparing the fractional error between LEDA distances and Tully, we obtain a $\sim 0.25$ log distance error for LEDA. 
TABLE 2

Summary of the Four Astronomical Catalogs Used to Develop the CBCG Catalog

\begin{tabular}{|c|c|c|c|c|}
\hline Catalog & $\begin{array}{c}\text { No. of } \\
\text { Galaxies }\end{array}$ & $\begin{array}{c}L_{10} \\
\left(10^{10} L_{B, \odot)}\right)\end{array}$ & $\begin{array}{l}\text { Fractional } \\
\text { Luminosity } \\
(\%)\end{array}$ & Reference \\
\hline 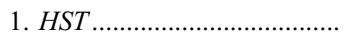 & 30 & 57.3 & 0.1 & Freedman et al. (2001) \\
\hline 2. Mateo & 18 & 0.4 & $<0.001$ & Mateo (1998) \\
\hline 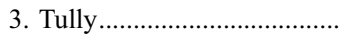 & 1968 & 2390 & 5.3 & B. Tully (2006, private communication) \\
\hline 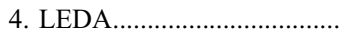 & 36741 & 42969.4 & 94.6 & Paturel et al. (2003) \\
\hline Total & 38757 & 45417.1 & 100.0 & \\
\hline
\end{tabular}

Nоте.-We report the number of galaxies for which the catalog was the primary reference and the fraction of the total CBCG catalog blue luminosity accounted for by those galaxies.

solar magnitude $M_{B, \odot}=5.48$ (Binney \& Tremaine 2000). We do not consider galaxies with luminosities less than $10^{-3} L_{10}$, because they do not contribute significantly to the total luminosity (see $\S 3)$.

The Mateo, Tully, and LEDA catalogs provide information on apparent $B$ magnitudes corrected for extinction. The galaxies in the HST Key Project catalog have only distance information, so for those we extract the corresponding apparent magnitude values $\left(m_{B}\right.$, corrected for internal and Galactic extinction) in the $B$ band from the Tully catalog to find $M_{B}$. Table 2 summarizes relevant properties of each of these catalogs and the fraction of the total luminosity within $100 \mathrm{Mpc}$ that each contributes.

The LEDA database quotes uncertainties in apparent magnitude. Figure 3 shows the distribution of LEDA assigned apparent magnitude variances for the galaxies in the CBCG catalog. The rms error is $\Delta m_{B}=0.42$. Galaxies from Tully's catalog have a smaller observational error $\Delta m_{B}=0.30$ (B. Tully 2006, private communication).

\section{COMPLETENESS}

Observations of faint galaxies are difficult even in the nearby universe, and lead to systematic incompleteness in galaxy catalogs. Studies of galaxy luminosity functions can provide insight into how many galaxies are missing from a catalog (and hence the corresponding blue luminosity). Using the CBCG catalog, we can generate a luminosity function $N(L, D)$ which is the number

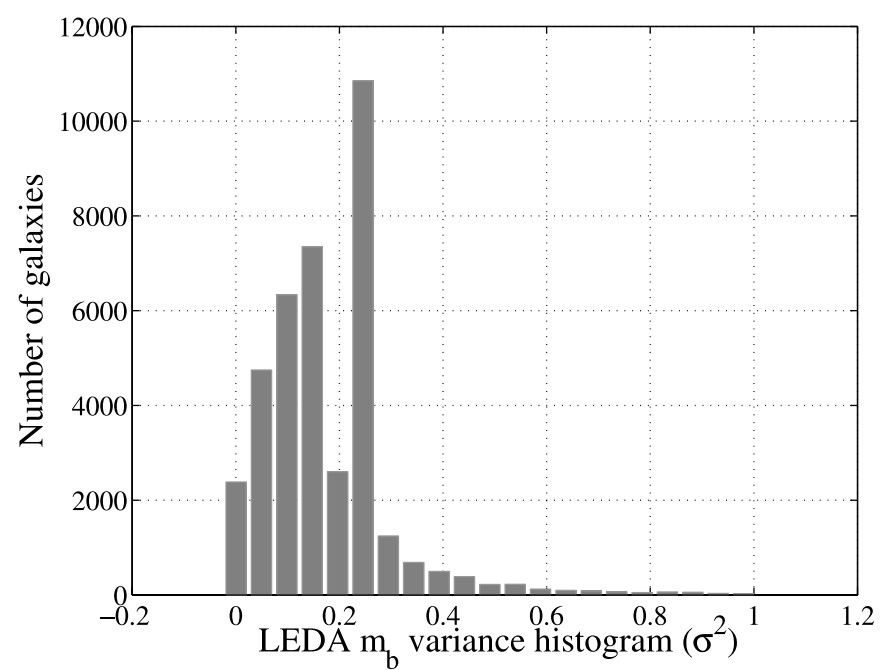

FIG. 3.-LEDA provides uncertainties in apparent magnitudes. The histogram above shows the $m_{b}$ variance distribution for each LEDA galaxy. The rms error is 0.42 . of galaxies with luminosities within a luminosity bin from $L$ to $L+\Delta L$ normalized to the spherical volume within radius $D$. Specifically, we write

$$
N(L, D) \Delta L=\left(\frac{3}{4 \pi D^{3}}\right) \sum_{j} l_{j}
$$

where

$$
l_{j}= \begin{cases}1 & \text { if }\left(L<L_{j}<L+\Delta L\right) \text { and }\left(D_{j}<D\right) \\ 0 & \text { otherwise }\end{cases}
$$

and the sum over $j$ runs through all the galaxies in the catalog. The quantities $L_{j}$ and $D_{j}$ are the luminosity and distance of each galaxy. Similarly, we can compute the luminosity function in terms of blue absolute magnitudes as a function of distance $N\left(M_{B}, D\right)$. The dashed and dot-dashed lines in Figure 4 show several realizations of $N\left(M_{B}, D\right)$ for different distances $D$ plotted as a function of $M_{B}$.

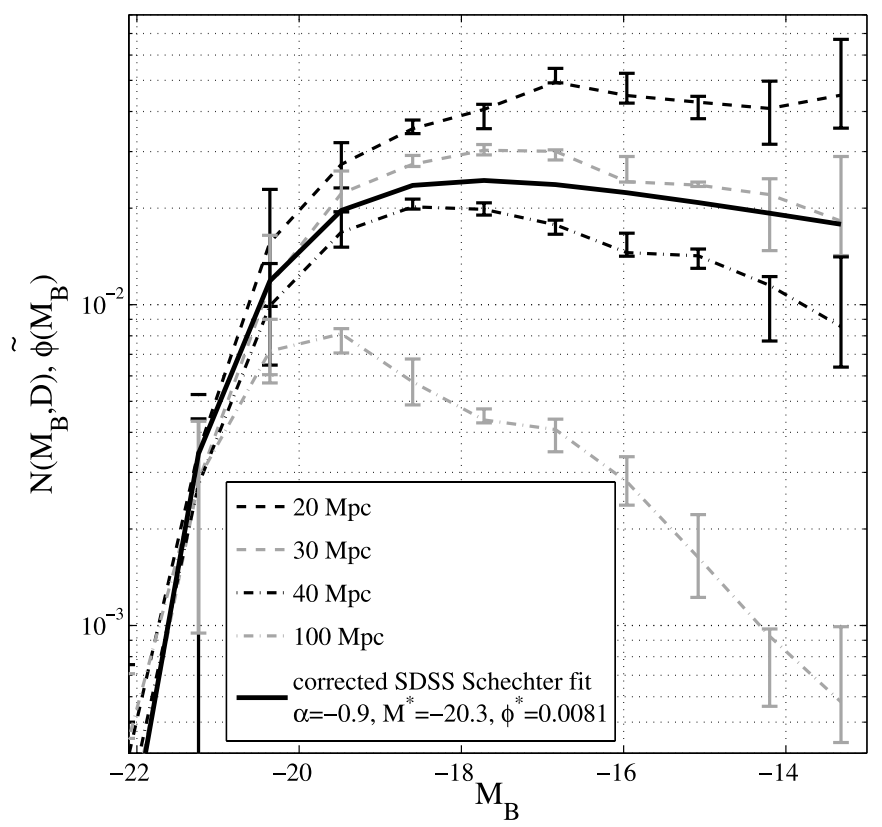

FIG. 4.-Luminosity function of the CBCG catalog at various distances (dashed and dot-dashed lines) and a Schechter function fit (solid line) given in eq. (3), based on Blanton et al. (2003). We compensate for the incompleteness of the CBCG catalog by applying an upward correction to the luminosity bins that are below the Schechter function fit (solid line), according to eqs. (4) and (6). Error bars are found by sliding the magnitudes of each galaxy according to the mean errors and recomputing the luminosity function. 
To estimate the degree of incompleteness in the CBCG catalog, we use an analytical Schechter galaxy luminosity function (Schechter 1976),

$$
\phi(L) d L=\phi^{*}\left(\frac{L}{L^{*}}\right)^{\alpha} \exp \left(\frac{-L}{L^{*}}\right) d\left(\frac{L}{L^{*}}\right),
$$

where $\phi(L) d L$ is the number density (number of galaxies per unit volume) within the luminosity interval $L$ and $L+d L, L^{*}$ is the luminosity at which the number of galaxies begins to fall off exponentially, $\alpha$ is a parameter which determines the slope at the faint end of the luminosity function, and $\phi^{*}$ is a normalization constant. In terms of (blue) absolute magnitudes, $M_{B}$, the Schechter function becomes

$$
\begin{aligned}
\tilde{\phi}\left(M_{B}\right) d M_{B}= & 0.92 \phi^{*} \exp \left[-10^{-0.4\left(M_{B}-M_{B}^{*}\right)}\right] \\
& \times\left[10^{-0.4\left(M_{B}-M_{B}^{*}\right)}\right]^{\alpha+1} d M_{B} .
\end{aligned}
$$

To estimate the total luminosity function, we use results from the Sloan Digital Sky Survey (SDSS) as reported by Blanton et al. (2003). Although the SDSS sky coverage is inadequate in R.A. and decl., it provides excellent coverage throughout our desired distance and beyond. We therefore use the green luminosity function Schechter fit given in Table 2 of Blanton et al. (2003) and convert it into the blue band using the expression given in Table 2 of Blanton \& Roweis (2007). Adopting a Hubble constant value of $73 \mathrm{~km} \mathrm{~s}^{-1} \mathrm{Mpc}^{-1}$ (Spergel et al. 2007) and correcting for reddening, ${ }^{14}$ the Schechter parameters are $\left(M_{B}^{*}, \tilde{\phi}^{*}, \alpha\right)=$ $(-20.3,0.0081,-0.9)$. The solid line in Figure 4 shows the Schechter function $\tilde{\phi}\left(M_{B}\right)$ derived from these values. Since this function is obtained from deep surveys, it does not account for the local overdensity of blue light coming primarily from the Virgo cluster. For distances up about to $30 \mathrm{Mpc}$, the $\mathrm{CBCG}$ catalog's luminosity function $N\left(M_{B}, D\right)$ exceeds $\tilde{\phi}\left(M_{B}\right)$.

We can now derive a completeness correction that arises at the faint end beyond about $30 \mathrm{Mpc}$, where the Schechter function exceeds the catalog $N\left(M_{B}, D\right)$. We integrate the CBCG galaxy catalog luminosity function $N(L, D)$ over $L$ and subtract it from the Schechter fit as a function of distance. Hence, the total corrected cumulative luminosity $L_{\text {total }}$ within a volume of radius $D$ is given by

$$
L_{\text {total }}(D)=L_{\mathrm{CBCG}}(D)+L_{\text {corr }}(D),
$$

where

$$
\begin{aligned}
& L_{\mathrm{CBCG}}(D)= \int_{0}^{D} d D^{\prime} \sum j L_{j} \delta\left(D^{\prime}-D_{j}\right) \\
& L_{\text {corr }}(D)=\frac{4 \pi}{3} D^{3} \int_{L_{\min }}^{L_{\max }} L d L \Theta[\phi(L)-N(L, D)] \\
& \times[\phi(L)-N(L, D)] .
\end{aligned}
$$

Here, the index $j$ runs through all galaxies in the catalog, $\delta$ is the Dirac delta function, $\Theta$ is the step function, and $\phi(L)$ is the adopted Schechter function (distance independent) assumed to represent the complete luminosity distribution. We note that $L_{\max }=52.481 L_{10}$ $\left(M_{B}=-23.83\right)$ is the maximum luminosity in the CBCG catalog, and we choose $L_{\min }=10^{-3} L_{10}\left(M_{B}=-12.98\right)$, because

\footnotetext{
14 We correct the value of $M_{B^{*}}$ to be consistent with the reddening correction described in $\S 3.1$.
}

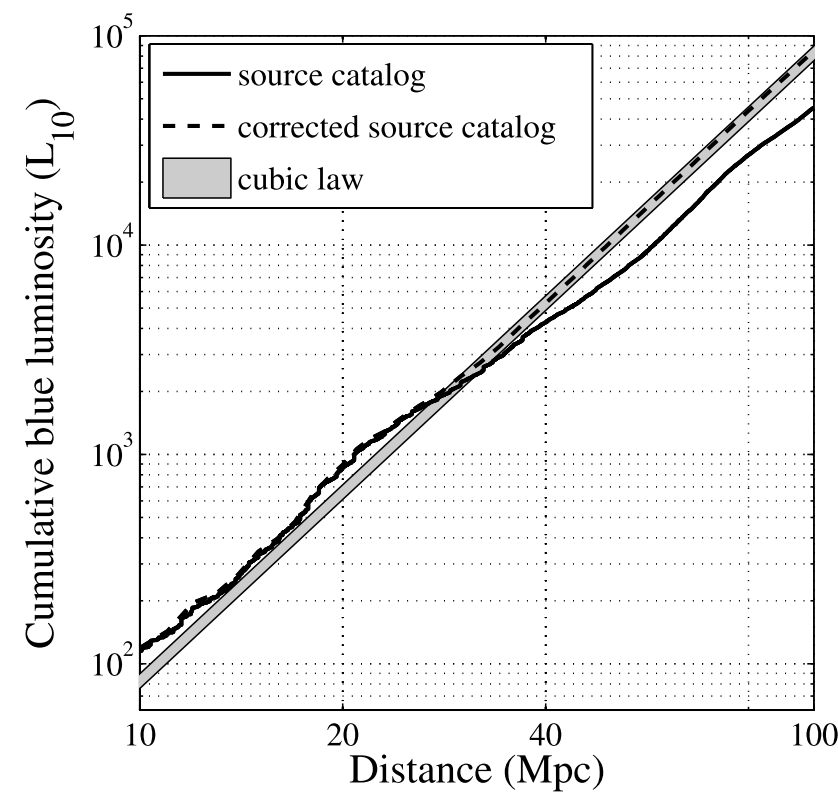

FIG. 5.-Cumulative luminosity as a function of distance from the CBCG catalog uncorrected for incompleteness (solid line), corrected for incompleteness (dashed line), and the cubic extrapolation from the assumed constant blue luminosity density corrected for extinction (gray-shaded region).

luminosities below this value do not contribute significantly to the net luminosity. The quantity $L_{\mathrm{CBCG}}$ in equations (4) and (5) is the uncorrected cumulative luminosity from the CBCG catalog; the quantity $L_{\text {corr }}$ is the completeness correction. Note that the completeness correction term is always zero or positive, regardless of the choice of Schechter function.

In Figure 5, we show the cumulative blue luminosity as a function of distance as obtained directly from the CBCG catalog (solid line), as well as with the completeness correction applied (dashed line). It is evident that the correction becomes significant at distances in excess of about $40 \mathrm{Mpc}$.

\subsection{Comparison with Other Results}

To compare our method of correcting for completeness with other methods, we consider the direct computation of a reddeningcorrected luminosity density based on Blanton et al. (2003), which could be used at large distances.

We adopt a blue luminosity density of $(1.98 \pm 0.16) \times$ $10^{-2} L_{10} \mathrm{Mpc}^{-3}$, calculated as follows:

1. The blue luminosity density, in terms of blue absolute magnitudes per cubic Mpc, is -14.98 locally (redshift $z=0$ ) and -15.17 for $z=0.1$ (see Table 10 of Blanton et al. 2003), for a standard cosmology with $\Omega_{M}=0.3$ and $\Omega_{\Lambda}=0.7$. We use $z=0.1$, so that the results will be valid for advanced detectors.

2 . We convert the $z=0.1$ blue magnitude density $(-15.17)$ to luminosity units $1.33 \times 10^{-2} L_{10} \mathrm{Mpc}^{-3}$ and assign systematic errors $(\simeq 10 \%)$ associated with the photometry to obtain a luminosity density of $(1.33 \pm 0.13) \times 10^{-2} L_{10} \mathrm{Mpc}^{-3}$.

3 . We also correct for processing of blue light and re-emission in the infrared (IR) following Phinney (1991) and Kalogera et al. (2001). We use the analysis of Saunders et al. (1990), upwardly correcting their far-IR $(40-100 \mu \mathrm{m})$ luminosity density by $30 \%$ to account for emission down to $12 \mu \mathrm{m}$ (Kalogera et al. 2001), and convert to $L_{10}$ to obtain an IR luminosity density of $L_{\mathrm{IR}}=$ $(0.65 \pm 0.1) \times 10^{-2} L_{10} \mathrm{Mpc}^{-3}$. 
4. Adding both luminosity densities above and accounting for the errors, we obtain a blue light luminosity density corrected for extinction equal to $(1.98 \pm 0.16) \times 10^{-2} L_{10} \mathrm{Mpc}^{-3}$.

We use this blue luminosity density and its uncertainty and plot the implied cumulative blue luminosity as a function of distance (cubic dependence) in Figure 5 (gray-shaded region). This uniform density distribution agrees well with the completenesscorrected luminosity given above.

We can compare our results for the cumulative blue luminosity as a function of distance to similar results obtained by Nutzman et al. (2004), especially their Figure 1. The results for the uncorrected catalog agree qualitatively. However, the catalog described here is more up-to-date than the one compiled by Nutzman et al. (2004), by virtue of the updates to LEDA and the inclusion of the current Tully catalog. The incompleteness correction derived here is also more physically and empirically motivated than the one constructed in that earlier paper. We note that the cumulative luminosity shown as the dashed line in their Figure 1 is too low by a factor of $4 \pi / 3$, due to a numerical error. In addition, their luminosity density is $\sim 25 \%$ lower than ours, as a result of our use of the more recent results presented by Blanton et al. (2003).

\section{COMPACT BINARY COALESCENCE RATE ESTIMATES}

For neutron star binaries, the observed binary pulsar sample can be used to predict the coalescence rate $\mathcal{R}_{\mathrm{MW}}$ in the Milky Way (Kim et al. 2004, 2006). The coalescence rate within a sphere of radius $D$ is then simply given by

$$
R=\mathcal{R}_{\mathrm{MW}}\left(\frac{L_{\text {total }}(D)}{L_{\mathrm{MW}}}\right)
$$

where $L_{\text {total }}(D)$ is the total blue luminosity within a distance $D$, and $L_{\mathrm{MW}}$ is the blue luminosity of the Milky way, $1.7 L_{10}$ (Kalogera et al. 2001). If the rate $R$ of a binary neutron star coalescence could be measured directly, it would provide an independent estimate of the rate of coalescence per unit of blue luminosity. Together, these two measurements would deepen our understanding of stellar and binary evolution. Furthermore, the current understanding of binary evolution and compact object formation leads us to anticipate the formation of black hole binaries that will merge within a Hubble time (e.g., Belczynski et al. 2002, 2007). Experiments like LIGO will provide a direct measure of the compact binary coalescence rate and will impose constraints on the theoretical models of stellar evolution and compact binary formation.

\subsection{Rate Estimates and Systematic Errors in Gravitational-Wave Searches}

In its simplest form, the rate estimate derived from a gravitationalwave experiment will take the form

$$
\mathcal{R}=\frac{\text { constant }}{T \mathcal{C}_{L}},
$$

where the constant depends on the precise outcome of the search and the statistical method used in arriving at the rate estimate, $\mathcal{C}_{L}$ is the cumulative blue luminosity observable within the search's sensitivity volume measured in $L_{10}$, and $T$ is the time analyzed in years. In general, the sensitivity volume is a complicated function that depends on the instrument and the gravitational waveforms searched for. Here, we focus on the influence of the host galaxy properties and the distribution of blue light with distance.

The gravitational-wave signal from a compact binary inspiral depends on a large number of parameters. It is convenient to split these parameters into two types for our discussion. Of particular interest here are the parameters that determine the location and orientation of the binary. We denote these collectively as $\lambda=$ $\{D, \alpha, \delta, \iota, \psi, t\}$, that is, the distance to the binary, its right ascension and declination, inclination angle relative to the line of sight, polarization angle of the waves, and the time when the binary is observed, respectively. Other parameters, including the masses and the spins, are denoted $\mu$. Recognizing that the spatial luminosity distribution can be written as

$$
L(\alpha, \delta, D)=\sum_{j} L_{j} \delta\left(\alpha_{j}-\alpha\right) \delta\left(\delta_{j}-\delta\right) \delta\left(D_{j}-D\right),
$$

we write the cumulative luminosity as

$$
\begin{aligned}
\mathcal{C}_{L}=\int & L(\alpha, \delta, D) p(\text { detection } \mid \boldsymbol{\mu}, \boldsymbol{\lambda}) \\
& \times p(\boldsymbol{\mu}) p(\iota) p(\psi) p(t) d \boldsymbol{\mu} d \boldsymbol{\lambda} .
\end{aligned}
$$

Assuming that binary coalescences are uniformly distributed in time, and their orientation is random, we take the corresponding prior probabilities: $p(\iota)=\sin (\iota) / 2, p(t)=1 /$ day, and $p(\psi)=1 / 2 \pi$.

Systematic errors associated with the derived rate estimates are naturally associated with the errors in cumulative luminosity $\mathcal{C}_{L}$. The two most relevant errors in the galaxy catalog are in apparent magnitude $m_{B}$ and distance $D$. Sky positions are known so precisely that small errors in R.A. and decl. do not change the detection probability of a particular binary in any significant way; for this reason, such errors are not included in the LIGO analyses (Abbott et al. 2007). The errors induced on the spatial luminosity function in equation (9) take the form (Fairhurst \& Brady 2007)

$$
\begin{aligned}
(L+\Delta L)(\alpha, \delta, D)= & \sum_{j} L_{j} 10^{-0.4 \Delta m_{B j}}\left(1+\frac{\Delta D_{j}}{D_{j}}\right)^{2} \\
& \delta\left(\alpha_{j}-\alpha\right) \delta\left(\delta_{j}-\delta\right) \delta\left(D_{j}+\Delta D_{j}-D\right) .
\end{aligned}
$$

\subsection{A Simplified Model for Estimating Expected Event Rates}

The sensitivity of a search for gravitational waves from compact binary coalescence is determined primarily by the amplitude of the waves at the detector. For a nonspinning binary (i.e., the spins of each compact object are much smaller than their general-relativistic maximum value of $m_{i}^{2}$ ) with given $\mu$, the amplitude is inversely proportional to the effective distance $D_{\text {eff }}$, defined as (Allen et al. 2005)

$$
D_{\text {eff }}=\frac{D}{\sqrt{F_{+}^{2}\left(1+\cos ^{2} \iota\right)^{2} / 4+F_{\times}^{2} \cos ^{2} \iota}},
$$

where $D$ is the physical distance to the binary, and $F_{+}$and $F_{\times}$are the response amplitudes of each polarization at the detector, which depend on the location of the binary system (Anderson et al. 2001),

$$
\begin{aligned}
& F_{+}=-\frac{1}{2}\left(1+\cos ^{2} \theta\right) \cos 2 \phi \cos 2 \psi-\cos \theta \cos 2 \phi \sin 2 \psi, \\
& F_{\times}=\frac{1}{2}\left(1+\cos ^{2} \theta\right) \cos 2 \phi \sin 2 \psi-\cos \theta \sin 2 \phi \cos 2 \psi .
\end{aligned}
$$

Here $\theta$ and $\phi$ are the spherical coordinates of the source defined with respect to the detector and, as before, $\iota$ and $\psi$ are the inclination 


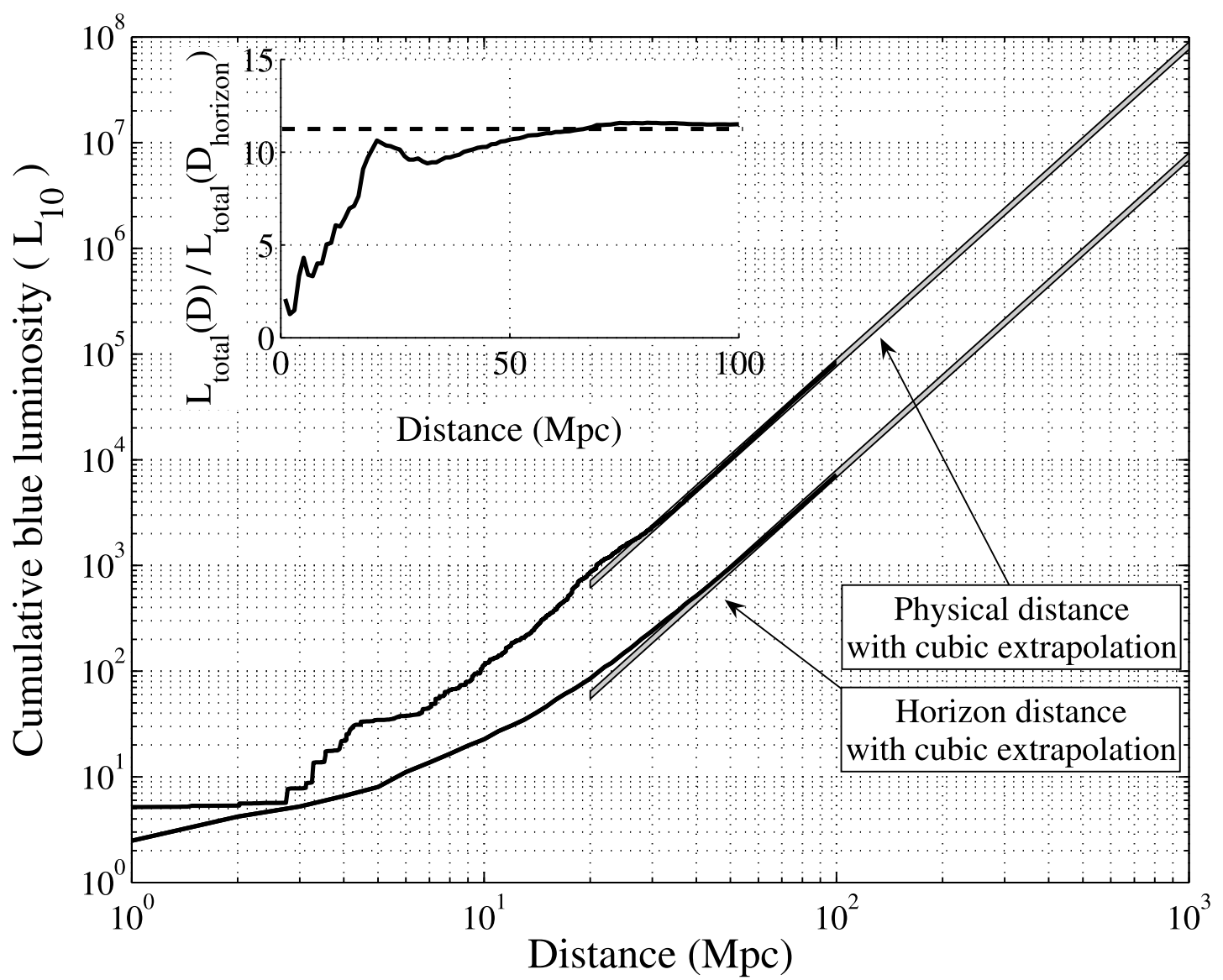

FIG. 6.-Cumulative luminosity as a function of physical distance (top line) and horizon distance (bottom line). The horizon distance $D_{\text {horizon }}$ is defined as the physical distance to an optimally oriented and located binary system that would be detected with a signal-to-noise ratio of 8 . (Instrumental sensitivity range is sometimes quoted in terms of the radius of a sphere with the same volume as the nonuniform region probed by the instrument; this sensitivity range $D_{s}$ is related to the horizon distance by $D_{s} \simeq D_{\text {horizon }} / \sqrt{5}$. The gray shaded lines are cubic extrapolations $(\S 3)$ derived for both cases. Given a LIGO horizon distance, one can immediately get the cumulative blue luminosity from the bottom curve. To obtain an approximate rate upper limit, one could calculate $\mathcal{R}_{90} \%\left[\mathrm{yr}^{-1} L_{10}^{-1}\right]=2.3 /\left(\mathcal{C}_{L} \times T\right)$ where $\mathcal{C}_{L}$ is taken from this plot at a given range in horizon distance. Inset: Ratio of the cumulative luminosity for the physical and horizon distance from the completeness-corrected $\mathrm{CBCG}$ catalog illustrates the nonuniform distribution at smaller ranges $(<20 \mathrm{Mpc})$ and asymptotes to the expected uniform distribution ratio (dashed line) for larger distances.

and polarization angles. Since $\theta$ and $\phi$ are detector dependent, the effective distance is different for geographically separated detectors that are not perfectly aligned, and for a fixed source location, changes as the Earth rotates through a sidereal day. In addition, the effective distance is always at least as large as the physical distance.

For simplicity in understanding the sensitivity of gravitational-wave searches, consider the case in which $\boldsymbol{\mu}$ is fixed, i.e., $p(\boldsymbol{\mu})=\delta(\boldsymbol{\mu}-\hat{\mu})$. For example, these might be the parameters appropriate to a neutron star binary. The sensitivity of a detector is given by its horizon distance, which is defined as the maximum effective distance that a neutron star binary system can be detected at a signal-to-noise ratio of 8 . Consider a search that can perfectly detect these binaries if they have an effective distance $D_{\text {eff }}<D_{\text {horizon }}$ at a particular detector. Then

$$
p(\operatorname{detection} \mid \hat{\mu}, \boldsymbol{\lambda})=\Theta\left(D_{\text {eff }}(\boldsymbol{\lambda})<D_{\text {horizon }}\right),
$$

and we can write

$$
\begin{aligned}
& \mathcal{C}_{L}\left(D_{\text {horizon }}\right)= \\
& \int L(\alpha, \delta, D) \Theta\left(D_{\text {eff }}(\lambda)<D_{\text {horizon }}\right) p(\iota) p(\psi) p(t) d \lambda .
\end{aligned}
$$

Thus, the cumulative blue luminosity accessible to such a detector is the blue luminosity within an effective distance sphere

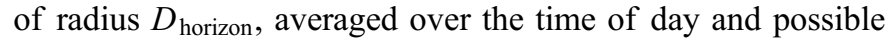
orientations of the binary. The lower curve in Figure 6 shows $\mathcal{C}_{L}\left(D_{\text {horizon }}\right)$.

Figure 6 also illustrates the significant difference between the cumulative luminosity $\mathcal{C}_{L}\left(D_{\text {eff }}\right)$ and total luminosity $L_{\text {total }}(D)$ at a given distance. If galaxies are distributed uniformly in space, the ratio between these is $\simeq 11.2$; this is the factor by which the detection rate would be reduced, and arises purely from the LIGO detector response, averaged over all possible source orientations with respect to the detector.

When estimating the rate based on gravitational-wave observations, one can marginalize over uncertainties (Fairhurst \& Brady 2007) in the galaxies' distances and apparent magnitudes. Specifically, by making use of the modified spatial distribution function equation (11) and the distributions for $\Delta D_{j}$ and $\Delta m_{B j}$ reported here, we can obtain a probability distribution for the cumulative luminosity $p\left(\mathcal{C}_{L} \mid \Delta m_{B j}, \Delta D_{j}\right)$ from equation (10). For each value of the cumulative luminosity, a probability distribution $p\left(R \mid \mathcal{C}_{L}\right)$ for the event rate can be calculated. Finally, the rate is marginalized over errors in the galaxy catalog by computing

$$
p(R)=\int d \mathcal{C}_{L} p\left(\mathcal{C}_{L} \mid \Delta m_{B j}, \Delta D_{j}\right) p\left(R \mid \mathcal{C}_{L}\right)
$$




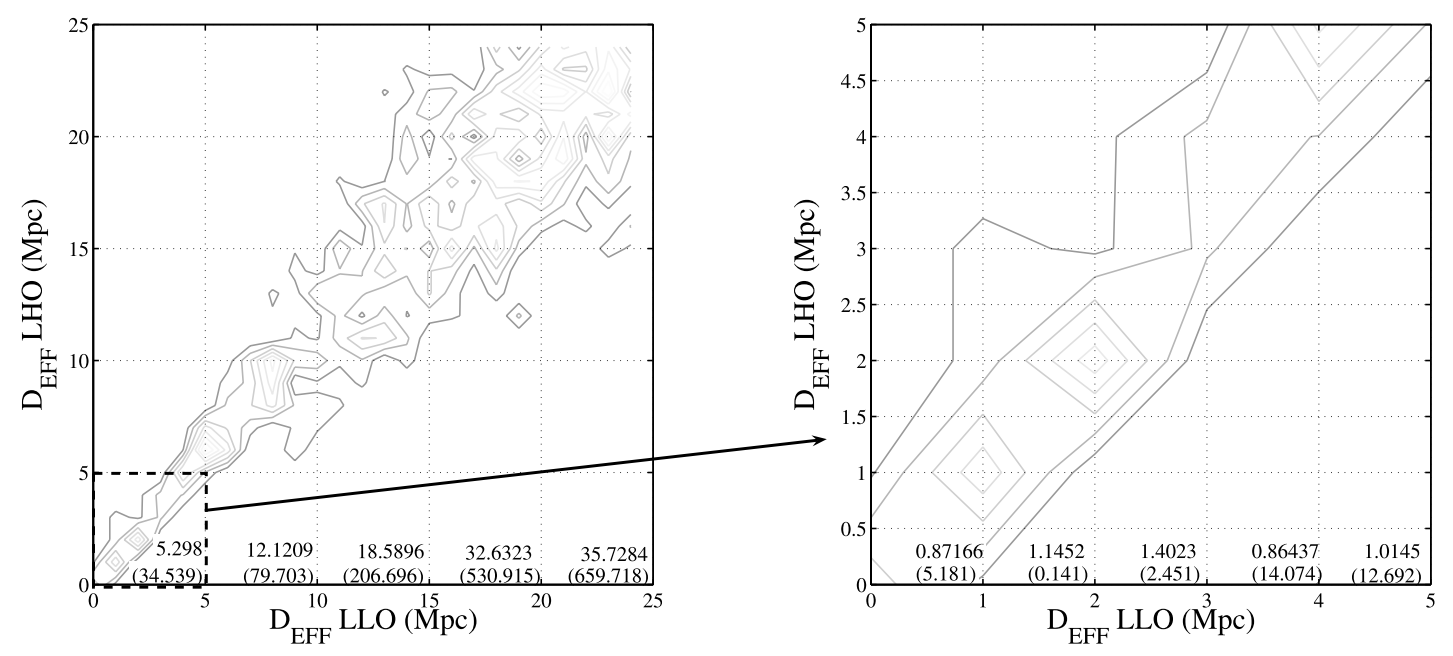

Fig. 7.-Luminosity contours per effective distance bin in the two LIGO sites. The effective distance to a source in one galaxy is different in the two detectors, and changes as a function of the sidereal day and also the orientation of the particular source. Since the effective distance is always larger than the real distance, the luminosity available within a given effective distance bin is considerably smaller than the luminosity within the physical distance bin. The upper horizontal numbers refer to the luminosity per bin in effective distance. The parenthetical lower numbers refer to the luminosity per physical distance bin. It is also possible to have a systematically different luminosity between the detectors, as indicated in the right panel zoom of the first $5 \mathrm{Mpc}$. The available luminosity within $5 \mathrm{Mpc}$ (mostly from Andromeda) is slightly better located for LLO, and therefore stretches the contours to higher effective distances for LHO. LIGO rate upper limits for searches with limited range thus depend on the nonuniformity of the Local Group.

This distribution is then used to obtain a rate interval or upper limit on the occurrence of binary coalescences in the universe.

While this approach provides a reasonable estimate of the observable blue light luminosity in a single detector, it does not provide the whole story. For example, the $16^{\circ}$ difference in latitude between the LIGO Observatories in Hanford, Washington and Livingston, Louisiana, implies that the $\mathcal{C}_{L}\left(D_{\text {horizon }}\right)$ depends on the site used. Figure 7 shows two-dimensional contours of this function.

Based on the galaxy catalog presented in this article, the cumulative blue luminosity $\mathcal{C}_{L}$, measured in $L_{10}$, accessible to a search with a given horizon distance sensitivity can be derived from Figure 6 and is tabulated in Table 3. We can combine the calculated cumulative blue luminosity with estimates of $\mathcal{R}$, the rate of binary mergers per $L_{10}$, to estimate the number of compact binary merger events $N$ detectable in a given LIGO search with an observation time $T$ :

$$
N=10^{-3}\left(\frac{\mathcal{R}}{L_{10}^{-1} \mathrm{Myr}^{-1}}\right)\left(\frac{\mathcal{C}_{L}}{10^{3} L_{10}}\right)\left(\frac{T}{\mathrm{yr}}\right)
$$

TABLE 3

Cumulative Blue Luminosity $\mathcal{C}_{L}\left(D_{\text {horizon }}\right)$ Accessible to a Search with Horizon Distance $D_{\text {horizon }}$

\begin{tabular}{|c|c|}
\hline $\begin{array}{l}D_{\text {horizon }} \\
(\mathrm{Mpc})\end{array}$ & $\begin{array}{c}\mathcal{C}_{L}\left(\mathcal{D}_{\text {horizon }}\right) \\
\left(L_{10}\right)\end{array}$ \\
\hline$\ldots$ & 23 \\
\hline ......................... & 85 \\
\hline 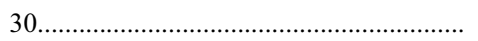 & 240 \\
\hline 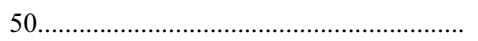 & 953 \\
\hline 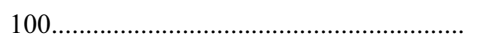 & 7200 \\
\hline 200 & 59200 \\
\hline $300 \ldots \ldots \ldots \ldots$ & 200000 \\
\hline 500 & 926000 \\
\hline
\end{tabular}

Note.-For $D_{\text {horizon }}>100 \mathrm{Mpc}$, the cumulative blue luminosity accessible to a search is given approximately by $\mathcal{C}_{L}\left(D_{\text {horizon }}\right) \approx 7.4 \times 10^{3}\left(D_{\text {horizon }} / 100 \mathrm{Mpc}\right)^{3}$.
If the horizon distance of a search is larger than $50 \mathrm{Mpc}$, we can use the following approximation, from a cubic law:

$$
N \approx 7.4 \times 10^{-3}\left(\frac{\mathcal{R}}{L_{10}^{-1} \mathrm{Myr}^{-1}}\right)\left(\frac{D_{\text {horizon }}}{100 \mathrm{Mpc}}\right)^{3}\left(\frac{T}{\mathrm{yr}}\right) .
$$

Estimated rates of binary neutron star (BNS) mergers in our Galaxy are based on the observed sample of binary pulsars. The rates depend on the Galactic distribution of compact objects. In Kalogera et al. (2004), the most recent reference estimating rates, the most likely Galactic rate for their reference model 6 is $83 \mathrm{Myr}^{-1}$, with a $95 \%$ confidence interval of $17-292 \mathrm{Myr}^{-1}$. The most likely rates for all the models used in Kalogera et al. (2004) are in the range 4-220 $\mathrm{Myr}^{-1}$ for the Milky Way. ${ }^{15}$

For the $4 \mathrm{~km}$ LIGO detectors currently operating, $D_{\text {horizon }} \approx$ $30 \mathrm{Mpc}$ for BNS. Thus, the predicted number of BNS events is in the range $N_{6} \approx 2-30 \times 10^{-3} \mathrm{yr}^{-1}$, with the most likely number being $N_{6} \approx 1 /(100 \mathrm{yr})$ (we use the subscript 6 to indicate that these rates use reference model 6 from Kalogera et al. 2004). A search that reaches twice the distance (such as enhanced LIGO) yields a most likely rate of $N_{6} \approx 1 /(10 \mathrm{yr})$, and a search 15 times more sensitive to the coalescences of binary systems than the current LIGO detectors (such as Advanced LIGO) would yield a most likely rate of $N_{6} \approx 40.0 \mathrm{yr}^{-1}$.

\section{CONCLUSION}

Whether one wishes to compute expected detection rates for LIGO searches, or to interpret LIGO searches as rate upper limits (or eventually detection rates), we require at the simplest level an accurate accounting of the total observable blue luminosity $\mathcal{C}_{L}$. As mentioned in the previous sections, a galaxy catalog complete with sky positions and distances is important for first

\footnotetext{
15 The rates quoted here are in units of rate per Milky Way per Myr; to get the rate per $L_{10}$, we divide by 1.7 , which is the estimated blue luminosity of the Milky way in $L_{10}$ units, assuming the blue absolute magnitude of the Milky Way to be -20.11 (Kalogera et al. 2001).
} 
generation LIGO detectors, because the blue luminosity is not uniformly distributed in the sky within the search range. An upper limit that takes into account the most up-to-date information on galaxy distributions can be obtained by accurately modeling the local overdense region. For searches with ranges well beyond current sensitivity, the universe is uniform, and rate estimates will depend primarily on accurate blue luminosity densities corrected for reddening. We have introduced a method to bridge the gap between the well-known nearby galaxy distribution and the expected long-range distribution through a completeness correction based on SDSS luminosity functions (Blanton et al. 2003).

This paper provides the most up-to-date accounting of nearby galaxies within $100 \mathrm{Mpc}$, as well as errors in the apparent magnitude (corrected for reddening) and distance, and demonstrates how the errors propagate into rate calculations. Astrophysical errors are a significant contribution to the eventual systematic error associated with coalescence rate upper limits (Fairhurst \& Brady 2007), and must be included. This paper provides a survey of the asymptotic and local uncertainty. Motivated by the use of effective distance to account for the antenna pattern of the LIGO detectors, we demonstrate the need to compute the average blue light luminosity within a given effective distance sphere. For ranges within $50 \mathrm{Mpc}$ there is a nontrivial relationship between cumulative blue luminosity within an effective distance sphere and within a physical distance sphere. Beyond $50 \mathrm{Mpc}$ the relationship is well-behaved, leading to the simple scaling for the number of detected events $N$ given in equation (19). We note that the catalog provided can also be used for other astronomical analysis of populations that scale with galaxy blue luminosity, such as the local Type II supernova rate or the rate of nearby soft gamma reapeater (SGR) bursts that show up as short GRBs.

We provide a sufficient description of our methods for others to apply new rate models to future LIGO data. Although this catalog will serve as a reference for current and future LIGO data analysis, we look forward to future work that may transcend the simple blue light rate normalization that we have discussed. One way to go beyond blue light rate normalization (necessary to ascertain the degree to which old stars contribute to present-day mergers) is with multiband photometry of nearby galaxies, which can reconstruct their mass, morphology, and metallicity-dependent star formation history. With this information in hand, LIGO detections could be applied more stringently to assess the relative contribution that progenitors of different ages provide to the present-day merger rate.

We would like to thank B. Tully for generously providing his most up-to-date nearby galaxies catalog in the preparation of this work. We acknowledge the use of the HyperLeda database (http:// leda.univ-lyon1.fr). We also thank P. Nutzman and the members of the LIGO Scientific Collaboration Compact Binary Coalescence group for many insightful discussions. This work has been supported in part by NSF grants PHY-0200852, PHY-0353111, PHY 03-26281, PHY 06-00953, PHY 06-53462, PHY-0355289, AST-0407070, a David and Lucile Packard Foundation Fellowship in Science and Engineering (V. K.), a Cottrell Scholar Award from the Research Corporation (P. R. B.), the Royal Society (S. F.), and the Center for Computation and Technology (R. K. K.). This work was also supported by the Center for Gravitational Wave Physics, which is supported by the National Science Foundation under cooperative agreement PHY 01-14375. LIGO was constructed by the California Institute of Technology and Massachusetts Institute of Technology with funding from the National Science Foundation, and operates under cooperative agreement PHY-0107417. This paper has LIGO document number LIGO-P070065-00-Z.

\section{REFERENCES}

Abbott, B., et al. 2007, preprint (arXiv:0704.3368)

Allen, B., Anderson, W. G., Brady, P. R., Brown, D. A., \& Creighton, J. D. E. 2005, preprint (gr-qc/0509116)

Anderson, W. G., Brady, P. R., Creighton, J. D., \& Flanagan, É. É. 2001, Phys. Rev. D, 63, 042003

Belczynski, K., Kalogera, V., \& Bulik, T. 2002, ApJ, 572, 407

Belczynski, K., Taam, R. E., Rantsiou, E., \& van der Sluys, M. V. 2007, preprint (astro-ph/0703131)

Binney, J., \& Tremaine, S. 2000, Galactic Dynamics (Princeton: Princeton Univ. Press)

Blanton, M. R., \& Roweis, S. 2007, AJ, 133, 734

Blanton, M. R., et al. 2003, ApJ, 592, 819

de Freitas Pacheco, J. A., Regimbau, T., Vincent, S., \& Spallicci, A. 2006, Int. J. Mod. Phys. D, 15, 235

Driver, P, S., \& Allen, D, P. 2007, ApJ, 657, L85

Fairhurst, S., \& Brady, P. 2007, preprint (arXiv:0707.2410)

Freedman, W., et al. 2001, ApJ, 553, 47

Kalogera, V., Narayan, R., Spergel, D. N., \& Taylor, J. H. 2001, ApJ, 556, 340

Kalogera, V., et al. 2004, ApJ, 601, L179; erratum 614, L137
Kim, C., Kalogera, V., \& Lorimer, D. R. 2006, preprint (astro-ph/0608280)

Kim, C., Kalogera, V., Lorimer, D. R., \& White, T. 2004, ApJ, 616, 1109

Lipunov, V. M., Nazin, S. N., Panchenko, I. E., Postnov, K. A., \& Prokhorov, M. E. 1995, A\&A, 298, 677

Mateo, M. 1998, ARA\&A, 36, 435

Nutzman, P., Kalogera, V., Finn, L. S., Hendrickson, C., \& Belczynski, K. 2004, ApJ, 612, 364

O’Leary, R., O’Shaughnessy, R., \& Rasio, F. A. 2007, Phys. Rev. D, 76, 061504

Paturel, G., Petit, C., Prugniel, Ph., Theureau, G., Rousseau, J., Brouty, M., Dubois, P., Cambrésy, L. 2003, A\&A, 412, 45

Phinney, E. S. 1991, ApJ, 380, L17

Portegies Zwart, S. F., \& McMillan, S. L. W. 2000, ApJ, 528, L17

Saunders, W., Rowan-Robinson, M., Lawrence, A., Efstathiou, G., Kaiser, N., Ellis, R. S., \& Frenk, C. S. 1990, MNRAS, 242, 318

Schechter, P. 1976, ApJ, 203, 297

Shaya, E., Peebles, P. J., \& Tully, B. 1995, ApJ, 454, 15

Spergel, D. N., et al. 2007, ApJS, 170, 377 